\title{
Inhibiting MicroRNA-29a Protects Myocardial Ischemia-Reperfusion Injury by Targeting SIRT1 and Suppressing Oxidative Stress and NLRP3-Mediated Pyroptosis Pathway
}

\author{
Shoukun Ding, ${ }^{1}$ Donghai Liu, ${ }^{1}$ Lixia Wang, ${ }^{1}$ Guanggong Wang, and Yaobin Zhu \\ Department of Cardiology, People's Hospital of Zhengzhou University, Zhengzhou, Henan (S.D., L.W., G.W.); Department of \\ Cardiovascular Surgery, First Affiliated Hospital of Zhengzhou University, Zhengzhou, Henan (D.L.); and Department of Pediatric \\ Heart Surgery, Beijing Children's Hospital, Beijing (Y.Z.), People's Republic of China
}

Received February 25, 2019; accepted August 19, 2019

\begin{abstract}
To investigate the effects of microRNA-29a (miR-29a) on myocardial ischemia-reperfusion (l/R) injury and its specific mechanisms, we used H9C2 myocardial cells to establish a myocardial ischemia model by hypoxia/reoxygenation $(\mathrm{H} / \mathrm{R})$, and microRNA$29 \mathrm{a}$ inhibitor was interfered. Annexin V/propidium iodide and flow cytometry were used to detect the effects of cell death. C57 mice were used to establish were used to establish the I/R injury model, and H\&E staining was used to detect pathologic damage to heart tissues. The expressions of miR-29a silent information regulator factor 2-related enzyme 1 (SIRT1) and nucleotide-binding oligomerization domain like receptor protein 3 (NLRP3), as well as pyroptosis-related proteins were determined by quantitative reverse-transcription polymerase chain reaction and Western blot analysis. The serum levels of 2-hydroxybutyrate dehydrogenase $(\mathrm{HBDH})$, lactate dehydrogenase-1 (LDH), creatine kinase $(\mathrm{CK})$, creatine kinase $\mathrm{MB}$ activity (CK-MB), IMA, and inflammatory factors in $\mathrm{l} / \mathrm{R}$ rats were significantly up-regulated. In the I/R group, the expression of miR-29a was significantly up-regulated while SIRT1 was remarkably down-regulated. Dual luciferase reporter
\end{abstract}

assay showed SIRT1 was a direct target of miR-29a. Inhibition of miR-29a significantly up-regulated the expression of peroxisome proliferator-activated receptor gamma coactivator- $1 \alpha /$ nuclear respiratory factor- 2 and endothelial nitric oxide synthase while remarkably down-regulating levels of inducible nitric oxide synthase and malondialdehyde in $\mathrm{I} / \mathrm{R}$. The oxidative stress that was induced by $\mathrm{I} / \mathrm{R}$ injury was also suppressed by inhibition of miR-29a. All these effects of miR-29a inhibition were reversed by small interfering SIRT1. The in vitro H/R results showed that NLRP3caspase-1-mediated pyroptosis was activated in $\mathrm{H} / \mathrm{R}$ but was significantly inhibited by the inhibition of miR-29a. Inhibition of miR-29a improved myocardial I/R injury by targeting SIRT1 through suppressing oxidative stress and NLRP3-mediated pyroptosis.

\section{SIGNIFICANCE STATEMENT}

In this study, we showed for the first time that miR-29a could improve myocardial I/R injury through inhibition of pyroptosis.

\section{Introduction}

The recovery of blood perfusion after myocardial ischemia results in more obvious and serious injury than before reperfusion, which leads to the decline of cardiac function and even endangers life (Hausenloy and Yellon, 2013). This phenomenon is called myocardial ischemia-reperfusion ( $\mathrm{I} / \mathrm{R})$ injury (Anaya-Prado et al., 2002). It has become an important factor affecting therapies for coronary heart disease, especially for acute myocardial infarction (Sanderson et al., 2013). Studies have found that myocardial I/R injury and apoptosis, oxygen free-radical release, intracellular calcium overload, myocardial energy metabolism disorder, inflammatory response,

\footnotetext{
${ }^{1}$ S.D., D.L., and L.W. contributed equally to this work.
} https://doi.org/10.1124/jpet.119.256982. and vascular endothelial cell injury (Piper et al., 2003; Quadri et al., 2005).

Micro RNA (miRNA) is an endogenous, noncoding, singlechain, small-molecule RNA composed of about 22 bases (Lewis et al., 2003). It can negatively regulate target gene expression at the posttranscriptional level by degrading the target gene or inhibiting its translation through complete or incomplete complementary pairing with the $3^{\prime}$ noncoding region of the target gene and can down-regulate the expression of corresponding proteins (Rupaimoole and Slack, 2017; Chou et al., 2018). MiRNA is abundantly expressed in cardiomyocytes and is involved in the process of cardiomyocyte apoptosis, arrhythmia, cardiac hypertrophy, and heart failure (Tang et al., 2009; Jayawardena et al., 2012).

Micro RNA 29a (miR-29a), an important part of miR-29 family, plays an important role in organisms (Roderburg et al.,

ABBREVIATIONS: CK, creatine kinase; CK-MB, creatine kinase MB activity; eNOS, endothelial nitric oxide synthase; HBDH, 2-hydroxybutyrate dehydrogenase; H/R, hypoxia/reoxygenation; IL, interleukin; iNOS, inducible nitric oxide synthase; I/R, ischemia-reperfusion; LDH, lactate dehydrogenase-1; MDA, malondialdehyde; miR-29a, micro-RNA 29a; miRNA, micro RNA; Mut, mutant; NC, negative control; NLRP3, nucleotidebinding oligomerization domain-like receptor protein 3; PCR, polymerase chain reaction; siRNA, small interfering RNA; SIRT1, silent information regulator factor 2-related enzyme 1; SOD, superoxide dismutase; TNF- $\alpha$, tumor necrosis factor- $\alpha$; WT, wild type. 
2011). Some studies have shown that the expression of microRNA-29a is up-regulated in myocardial I/R injury and that down-regulated expression of microRNA-29a can protect the heart from I/R injury. The underlying mechanism remains unclear (Sassi et al., 2017).

Silent information regulator factor 2 related enzyme 1 (SIRT1) is a deacetylase-dependent on nicotinamide adenine dinucleotide (Fusco et al., 2012). It plays an important role in inhibiting apoptosis, alleviating inflammatory reaction, protecting mitochondrial function, and resisting oxidative stress, but the specific mechanism is still unclear (Corpas et al., 2017). SIRT1 has been reported to be involved in the development of I/R injury and also could improve ischemic injury in the brain (Hattori et al., 2015). However, no studies have focused on the relationship of miR-29a and SIRT1 with myocardial I/R injury or oxidative stress and apoptosis. Our study investigated the role of the miR-29a/SIRT1 axis in myocardial I/R injury.

\section{Methods and Materials}

Animals and Treatment. A total of 30 C57BL/J6 mice (8 weeks, 20-30 g) were obtained from the animal center of the People's Hospital of Zhengzhou. All animals were kept in a light-controlled room under a 12-hour light/dark cycle and controlled temperature $\left(23-25^{\circ} \mathrm{C}\right)$, and they had free access to food and water according to the U.S. National Research Council's Guide for the Care and Use of Laboratory Animals. The research was approved by the Institutional Animal Care Committee at the People's Hospital of Zhengzhou.

The left anterior descending coronary artery ligation model was used to establish our myocardial I/R model (Han et al., 2014). The occlusion was confirmed by balancing of the left ventricular myocardium below the suture. Mice were subjected to 45 minutes of transitory ligation followed by 3 hours of reperfusion. The control group only received a sham operation that used the same procedure except for the placement of the ligature. At 72 hours after surgery, the animals were sacrificed before experiments. The infarction volume was measured by staining with 2,3,5-triphenyltetrazolium chloride (Sigma-Aldrich, St. Louis, MO) and calculated with ImageJ software (U.S. National Institutes of Health, Bethesda, MD). H\&E staining was used for the histologic analysis of myocardial tissues.

Cell Culture and Transfection. H9c2 cardiomyocytes (American Type Culture Collection, Manassas, VA) were seeded on 96-well plates and incubated with 10\% FBS Dulbecco's modified Eagle's medium (Sigma-Aldrich $\mathrm{Co}$ ) at $37^{\circ} \mathrm{C}, 5 \% \mathrm{CO}_{2}$. To establish the hypoxia/ reoxygenation $(\mathrm{H} / \mathrm{R})$ model, we cultured the cells in a $2.3 \% \mathrm{O}_{2}$, $5 \% \mathrm{CO}_{2}, 92.7 \% \mathrm{~N}_{2}$ hypoxic environment for 2 hours, and then cultured them at $37^{\circ} \mathrm{C} 5 \% \mathrm{CO}_{2}$ for 4 hours. Then the cells were transfected with miR-29a inhibitor or negative control (NC), as well as small interfering (siRNA) of SIRT1 (si-SIRT1) or si-NC (all above were purchased from GeneChem Corp., Shanghai, People's Republic of China) using the lipo6000 reagent (Beyotime Biotechnology, People's Republic of China) according to the manufacturer's instructions.

Quantitative Reverse-Transcription Polymerase Chain Reaction. Expression of miR-29a and SIRT1 was detected by quantitative reverse-transcription polymerase chain reaction (PCR). RNA was extracted with the TRIzol (Takara Biochemicals, Otsu, Japan) experimental method. The DNA was retrieved by stem-loop primers. Real-time PCR was performed after the DNA has been obtained. We designed a stem-loop reverse-transcription primer for MIR-29a: 5'-GTC GTATCCAGTGCAGGGGTCCGAGTATTCGCACTGGATACGACT AACCG-3', and a reverse transcription primer for U6: 5' -AACGCTTCA CGAATTTGCGGT-3', which was synthesized by GeneChem Corp.

The PCR reactions were conducted using ABI 7500 Fast Fluorescent Quantitative PCR instrument (Applied Biosystems, Foster City, CA), and the SYBR-green I dye method was used to perform the experiment.
The upstream and downstream primers of micro RNA miR-29a and internal reference gene $\mathrm{U} 6$ are to be shown in Table 1. The relative RNA levels were calculated by the $2^{-\Delta \Delta \mathrm{Cq}}$ method. Glyceraldehyde3-phosphate dehydrogenase (GAPDH) was used as an internal control.

Western Blot Analysis. The expression of SIRT1, nucleotidebinding oligomerization domain-like receptor protein 3 (NLRP3), caspase-1, interleukin-1 $\beta$ (IL- $1 \beta$ ), and the related pathways of endothelial nitric oxide synthase (eNOS) oxidative stress were detected by Western blot analysis. The treated cells were collected, centrifuged, and washed with PBS. Protein lysate, the protease inhibitor phenylmethylsulfonyl fluoride, and phosphatase inhibitor were added after washing. The cells were placed on ice for 40 minutes and centrifuged for 15 minutes at $4^{\circ} \mathrm{C} 12,000 \mathrm{~g}$. The supernatant was added to the SDS buffer and boiled in water for 5 minutes to make the protein sample.

After 10\% SDS-PAGE electrophoresis, the protein samples were transferred to polyvinylidene fluoride membranes at $300 \mathrm{~mA}$ for 90 minutes and sealed with 5\% skimmed milk powder at room temperature for 2 hours. The samples were then incubated with a primary antibody at $4^{\circ} \mathrm{C}$ overnight, followed by incubation with a corresponding secondary antibody at $37^{\circ} \mathrm{C}$ for 45 minutes.

The films were scanned using a Super Signal West Pico Chemiluminescent Substrate kit (Pierce/Thermo Fisher Scientific, Waltham, MA). The relative protein expression was quantified using Image-Pro Plus software (version 6.0; Media Cybernetics, Rockville, MD).

Dual Luciferase Assay. The binding of miR-29a to SIRT1 was detected by double luciferase assay. Bioinformatic prediction between miR-29a and SIRT1 was obtained from the software Targetscan 7.2 (http://www.targetscan.org; Whitehead Institute for Biomedical Research, Cambridge, MA). Briefly, the wild-type (WT) SIRT1 3' untranslated region or mutant (Mut) was amplified and subcloned into a pGL4.10 luciferase reporter vector. The cells were then cotransfected with either the vectors and the miR-29a mimics or NC using the Lipo6000 reagent. Luciferase assays were performed 48 hours after transfection using a Bright-Glo LuciferaseAssay System (Promega, Madison, WI). The luciferase activity was normalized to values of Renilla luciferase activity.

Detection of Cell Death. For the apoptosis analysis, briefly, the cells were stained with Annexin V/propidium iodide double-staining kit (BD Biosciences, Woburn, MA) strictly according to the manufacturer's introductions. Cell apoptosis was measured by flow cytometry (BD Biosciences).

Measurement of Serum and Cell Supernatant Factor Levels. Commercial ELISA kits (all purchased from Abcam, Cambridge, MA) were used to detect the serum and cell supernatant levels of inflammatory factors IL-1, IL-6, and tumor necrosis factor- $\alpha$ (TNF- $\alpha$ ) according to manufacturer's instructions. Levels of oxidative factors malondialdehyde (MDA) and superoxide dismutase (SOD) were measured using the corresponding kits (Nanjing Jiancheng Bio-Technology, Nanjing, People's Republic of China). Myocardial enzymes of 2-hydroxybutyrate dehydrogenase (HBDH), lactate dehydrogenase-1 (LDH), creatine kinase (CK), creatine kinase MB activity (CK-MB), and IMA were measured using an automatic biochemical analyzer (Olympus5400; Olympus, Tokyo, Japan).

Statistical Analysis. All data were analyzed by SPSS 22.0 software (IBM, Armonk, NY). The measurement data are all expressed as mean \pm S.D. The independent samples $t$ test was used to measure the data between two groups. Comparisons among three or more groups were conducted using one-way ANOVA followed by Tukey post hoc test. $P<0.05$ was considered statistically significant.

\section{Results}

Effects of Myocardial Ischemia Reperfusion on Myocardial Injury, Myocardial Enzymes, and Inflammatory Factors. First, we investigated effects of myocardial I/R injury on myocardial enzymes and inflammatory factors. 
TABLE 1

Primers used in quantitative reverse-transcription polymerase chain reaction

\begin{tabular}{ll}
\hline \multicolumn{1}{c}{ Primer Name } & \multicolumn{1}{c}{ Sequence $\left(5^{\prime}-3^{\prime}\right)$} \\
\hline miR-29a Upstream sequence & GCGGCGGTAGCACCATCTGAAATC \\
miR-29a Downstream sequence & GTGCAGGGTCCGAGGT \\
U6 upstream sequence & CTCGCTTCGGCAGCACA \\
U6 downstream sequence & AACGCTTCACGAATTTGCGT \\
SIRT1 upstream sequence & TTCAGGTCAAGGGATGGTATTT \\
SIRT1 downstream sequence & TGTTCCAGCGTGTCTATGTTCT \\
Actin upstream sequence & CTGGGACGACATGGAGAAAA \\
Actin downstream sequence & AAGGAAGGCTGGAAGAGTGC \\
\hline
\end{tabular}

As shown in Fig. 1, the infraction size was significantly higher in I/R mice $(P<0.05)$. H\&E staining also showed obvious myocardial injury in the $\mathrm{I} / \mathrm{R}$ group, suggesting the successful establishment of the I/R model. The serum levels of all myocardial enzymes-HBDH, LDH, CK, CK-MB and IMA-were statistically significantly elevated in the I/R mice $(P<0.05)$. We found similar results for the inflammatory factors IL-1, IL-6, and TNF- $\alpha$, which were remarkably up-regulated in the I/R mice $(P<0.05)$.
Myocardial I/R Up-Regulated miR-29a and Activated Oxidative Stress and Pyroptosis in Mice Myocardial Tissues. We determined the expression of miR-29a and SIRT1 in the myocardial tissues of the I/R and control mice. Our results showed the expression of miR-29a was significantly up-regulated in the $\mathrm{I} / \mathrm{R}$ group $(P<0.05$, Fig. 2). In addition, both the protein and mRNA levels of SIRT1 were remarkably down-regulated in the I/R mice $(P<0.05)$.

For oxidative stress, the levels of eNOS were significantly decreased, while inducible nitric oxide synthase (iNOS) was dramatically increased in the myocardial tissues of the $\mathrm{I} / \mathrm{R}$ mice $(P<0.05)$. Serum MDA was also elevated while SOD was down-regulated in the I/R mice $(P<0.05)$.

We also determined the levels of the pyroptosis-related proteins NLRP3, caspase- 1 , and IL- $1 \beta$. We observed that the protein levels of NLRP3, caspase- 1 , and IL- $1 \beta$ were all significantly up-regulated in the $\mathrm{I} / \mathrm{R}$ mice $(P<0.05)$. These results indicated that myocardial I/R up-regulated miR-29a, down-regulated SIRT1, and activated oxidative stress and pyroptosis.

miR-29a Directly Targeted SIRT1 in H9c2 Cells. To confirm binding between miR-29a and SIRT1 in H9c2 cells, we
A
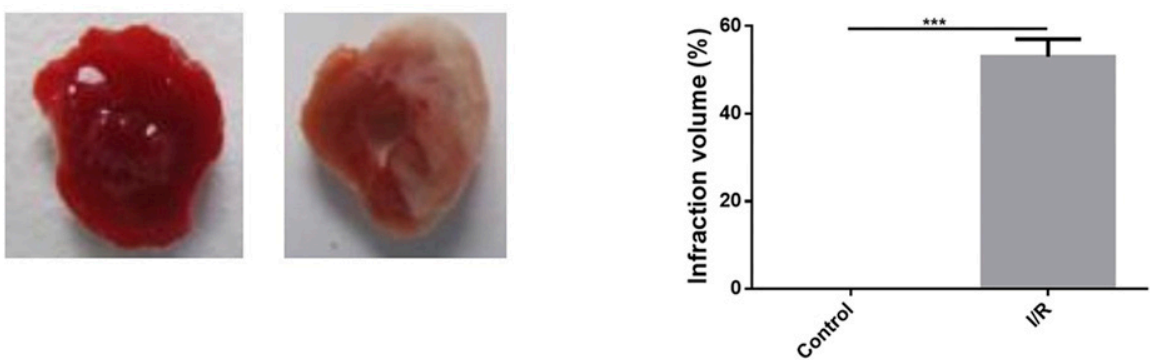

B
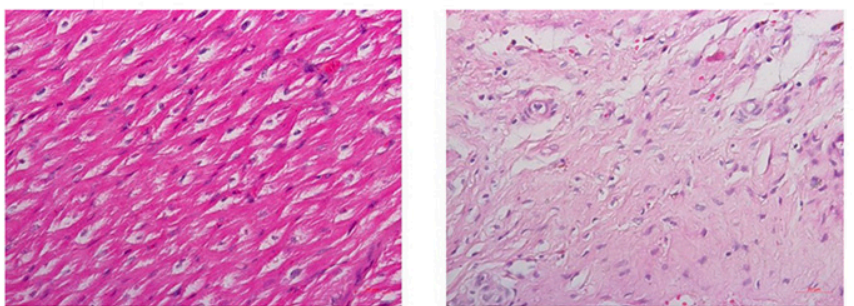

C
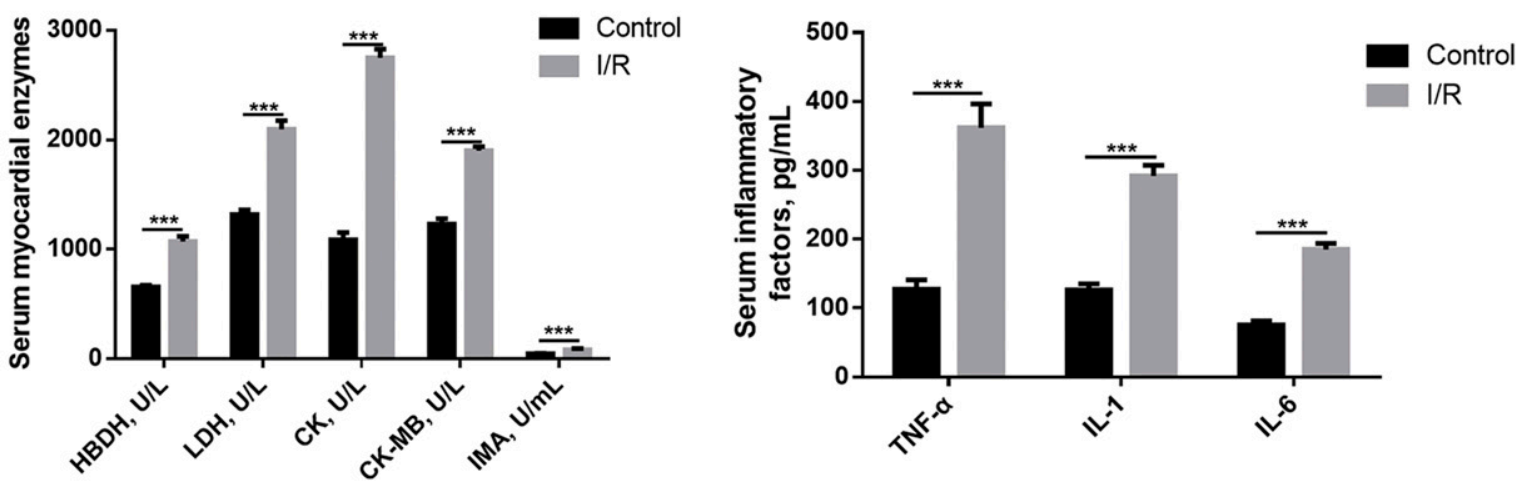

Fig. 1. Effects of myocardial ischemia reperfusion on myocardial injury, myocardial enzymes, and inflammatory factors. (A) 2,3,5-Triphenyltetrazolium chloride staining and infraction volume of different mice. (B) H\&E staining of different mice. (C) Serum levels of myocardial enzymes and inflammatory factors in different groups of mice. ${ }^{* * *} P<0.001$. 

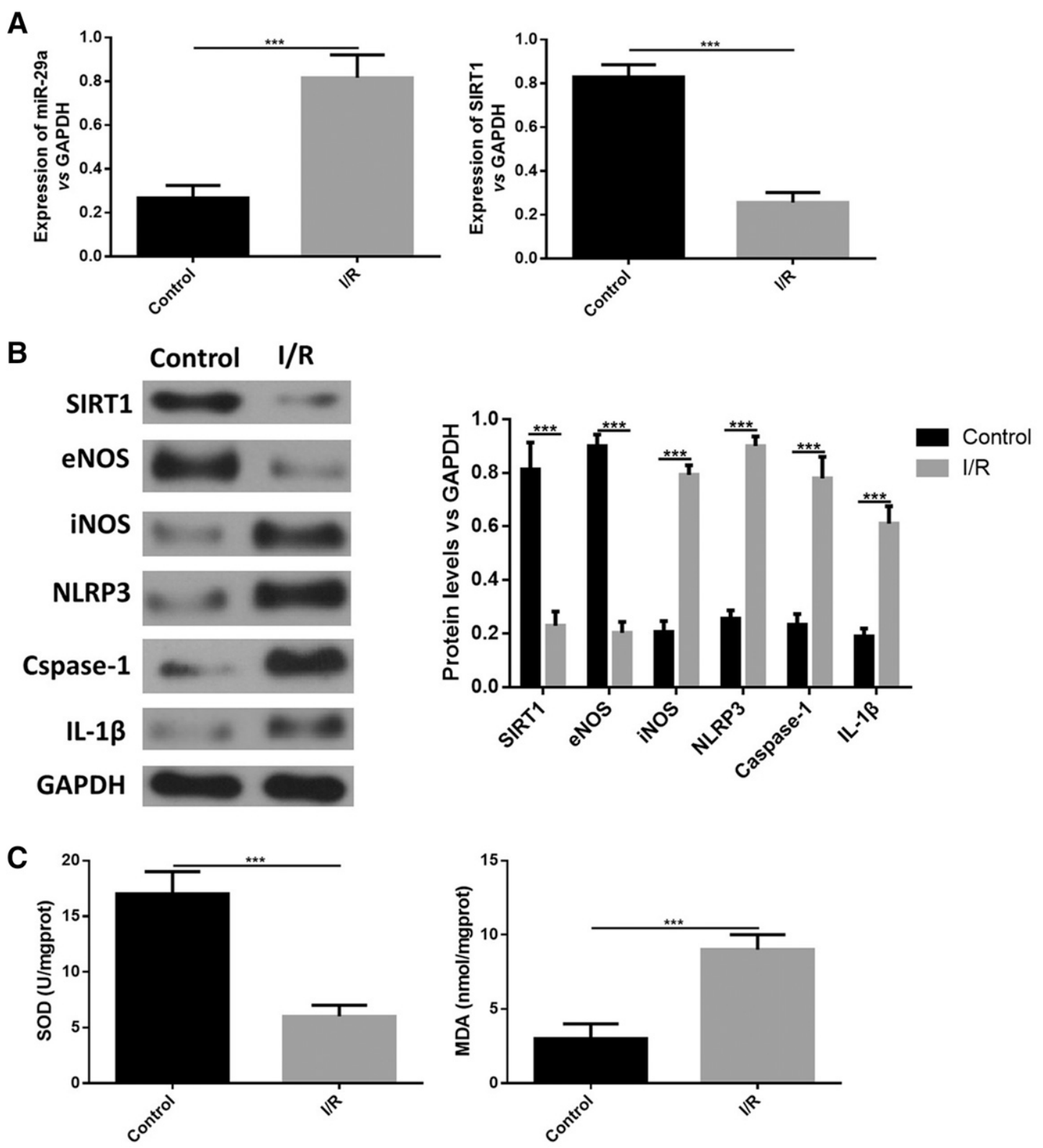

Fig. 2. Myocardial I/R up-regulated miR-29a and activated oxidative stress and pyroptosis in mice myocardial tissues. (A) Expression of miR-29a and SIRT1 in mice tissues of I/R and control. (B) Protein expression of SIRT1, eNOS, iNOS, NLRP3, caspase-1, and IL-1 $\beta$ in I/R and control mice. (C) SOD and MDA levels in $\mathrm{I} / \mathrm{R}$ and control mice. ${ }^{*} * P<0.001$.

conducted a dual luciferase assay. The results showed the luciferase activity to be significantly lower when the cells were transfected with miR-29a mimics in WT-SIRT1 $(P<0.05$, Fig. 3), but no significant change was found with Mut-SIRT1. Furthermore, when transfected with miR-29a mimics, the expression of SIRT1 was significantly down-regulated but inhibition of miR-29a remarkably increased the SIRT1 level compared with the NC $(P<0.05)$. All these results demonstrated miR-29a directly targeted and negatively regulated SIRT1.

Inhibition of miR-29a Suppressed H/R-Induced Oxidative Stress in H9c2 Cells. We used an H/R model to simulate in vitro I/R conditions. As shown in Fig. 4, treatment of $\mathrm{H} / \mathrm{R}$ significantly up-regulated the expression of miR-29a but down-regulated the SIRT1 level $(P<0.05)$. Transfection of miR-29a inhibitor remarkably down- regulated the expression of miR-29a and up-regulated the expression of SIRT1; si-SIRT1 significantly downregulated the SIRT1 levels, which was elevated by inhibition of miR-29a $(P<0.05)$. The expression of eNOS was significantly increased in $\mathrm{H} / \mathrm{R}$ cells, and inhibition of miR-29a significantly inhibited the eNOS level, which was reversed by inhibition of SIRT1.

Opposite results were observed for iNOS. Meanwhile, levels of MDA were significantly increased while SOD was significantly decreased in $\mathrm{H} / \mathrm{R}$ cells $(P<0.05)$. The inhibition of miR29a significantly decreased the MDA level and increased the SOD level, but inhibition of SIRT1 remarkably reversed these effects $(P<0.05)$.

All these results suggest that inhibition of miR-29a suppressed oxidative stress in H/R H9c2 cells through regulation of SIRT1. 
A

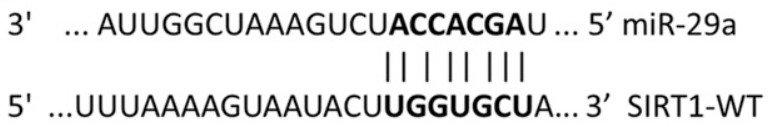

-UACCACGAA- SIRT1-MUT
B

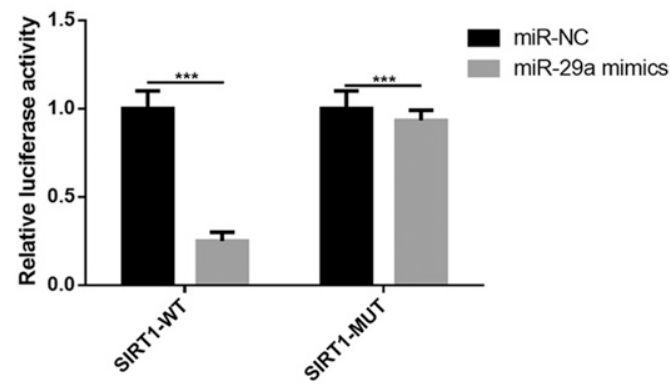

C
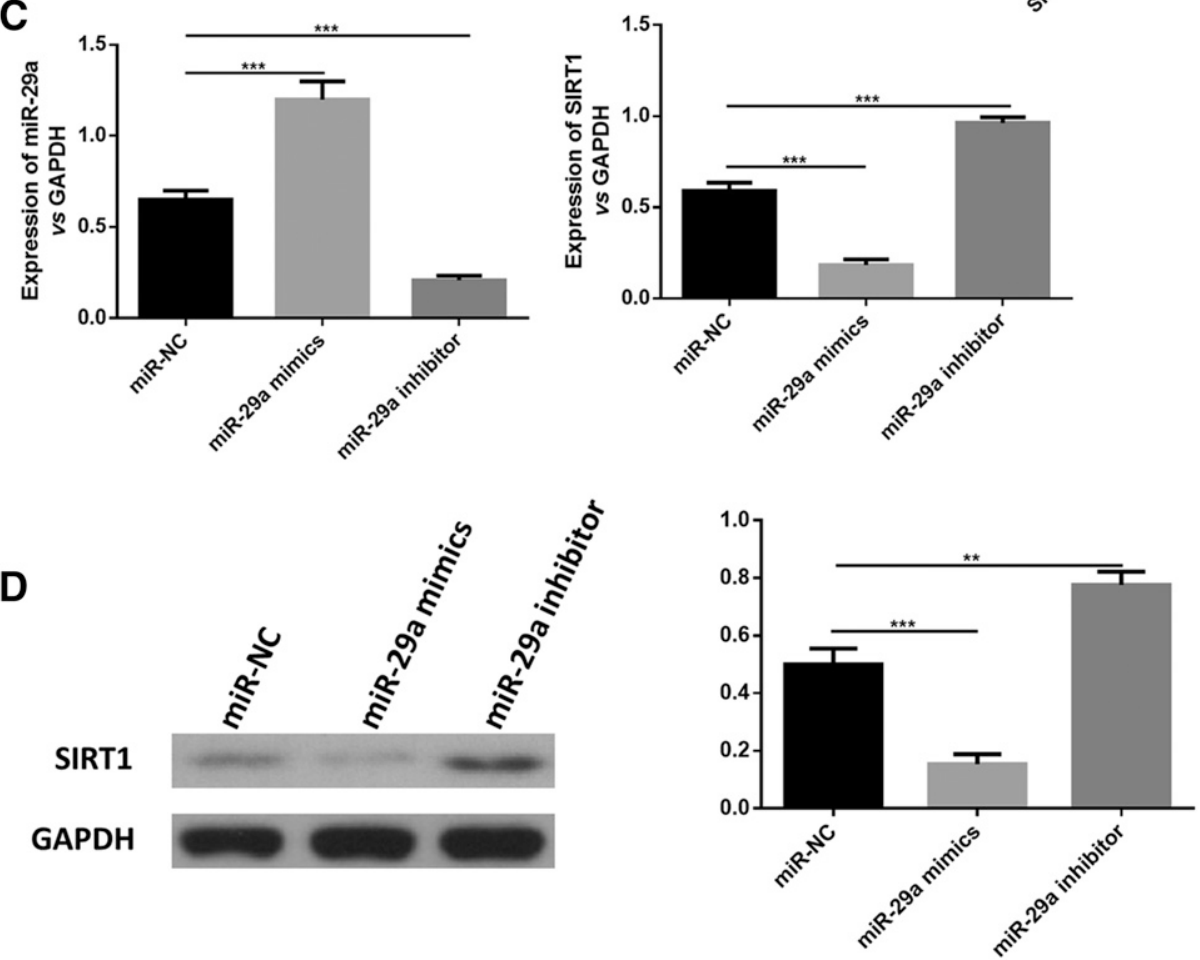

Fig. 3. Micro RNA 29a directly targeted SIRT1 in H9c2 cells. (A) Predicted region of miR-29a and SIRT1. (B) Relative Renilla luciferase activity for SIRT1-WT and SIRT1-Mut. (C) Expression of miR-29a and SIRT1 in cells transfected with miR-29a mimics, inhibitor, or NC. (D) Protein levels of SIRT1 in cells transfected with miR-29a mimics, inhibitor, or NC. ${ }^{* * *} P<0.001 ; * * P<0.01$.

Inhibition of miR-29a Suppressed H/R-Induced Pyroptosis in H9c2 Cells. Finally, we detected pyroptosisrelated proteins and inflammatory factors in our $\mathrm{H} / \mathrm{R}$ model. The results showed that treatment of $\mathrm{H} / \mathrm{R}$ significantly elevated all pyroptosis-related proteins of NLRP3, caspase-1, and IL- $1 \beta$, as well as levels of IL-1, IL-6, and TNF- $\alpha$. Inhibition of SIRT1 dramatically reversed these effects $(P<0.05$, Fig. 5$)$.

We also examined cell apoptosis. The treatment of $\mathrm{H} / \mathrm{R}$ significantly enhanced the apoptosis rate, but inhibition of miR-29a dramatically suppressed apoptosis, which was also reversed by inhibition of SIRT1 $(P<0.05)$, suggesting that inhibition of miR-29a suppressed H/R-induced pyroptosis in $\mathrm{H} 9 \mathrm{c} 2$ cells through regulation of SIRT1.

\section{Discussion}

Myocardial I/R injury can be seen in open heart surgery, coronary artery bypass grafting, coronary angioplasty, thrombolytic surgery, and in the sudden increase in blood flow of the medial myocardial branch. With the increasing incidence of myocardial infarction and the extensive development of early coronary artery recanalization therapy, myocardial I/R injury has become a common pathophysiological phenomenon in clinical situations. How to alleviate I/R injury is an urgent problem to be solved.

Despite numerous studies on I/R injury, the underlying mechanisms remain unclear. Up to now, no studies have focused on the effects of miR-29a on oxidative stress and NLRP3-mediated pyroptosis in myocardial I/R injury. In our present study, we demonstrated for the first time that inhibition of miR-29a improved myocardial I/R injury by targeting SIRT1 through suppressing oxidative stress and NLRP3-mediated pyroptosis.

MiR-29a has been proven to be involved in many diseases, including I/R injury. Duan et al. (2017) reported that miR-29a was involved in protective remote ischemic preconditioning in an I/R rat model. These investigators found that reducing miR-29a levels may produce a protective effect in liver I/R injury. Sassi et al. (2017) also demonstrated that inhibition of miR-29a could prevent cardiac hypertrophy and fibrosis and improve cardiac function. It also has been demonstrated that miR-29a is up-regulated after myocardial I/R, and that increased miR-29a may promote cell apoptosis (Wang et al., 2015). 


\section{A}
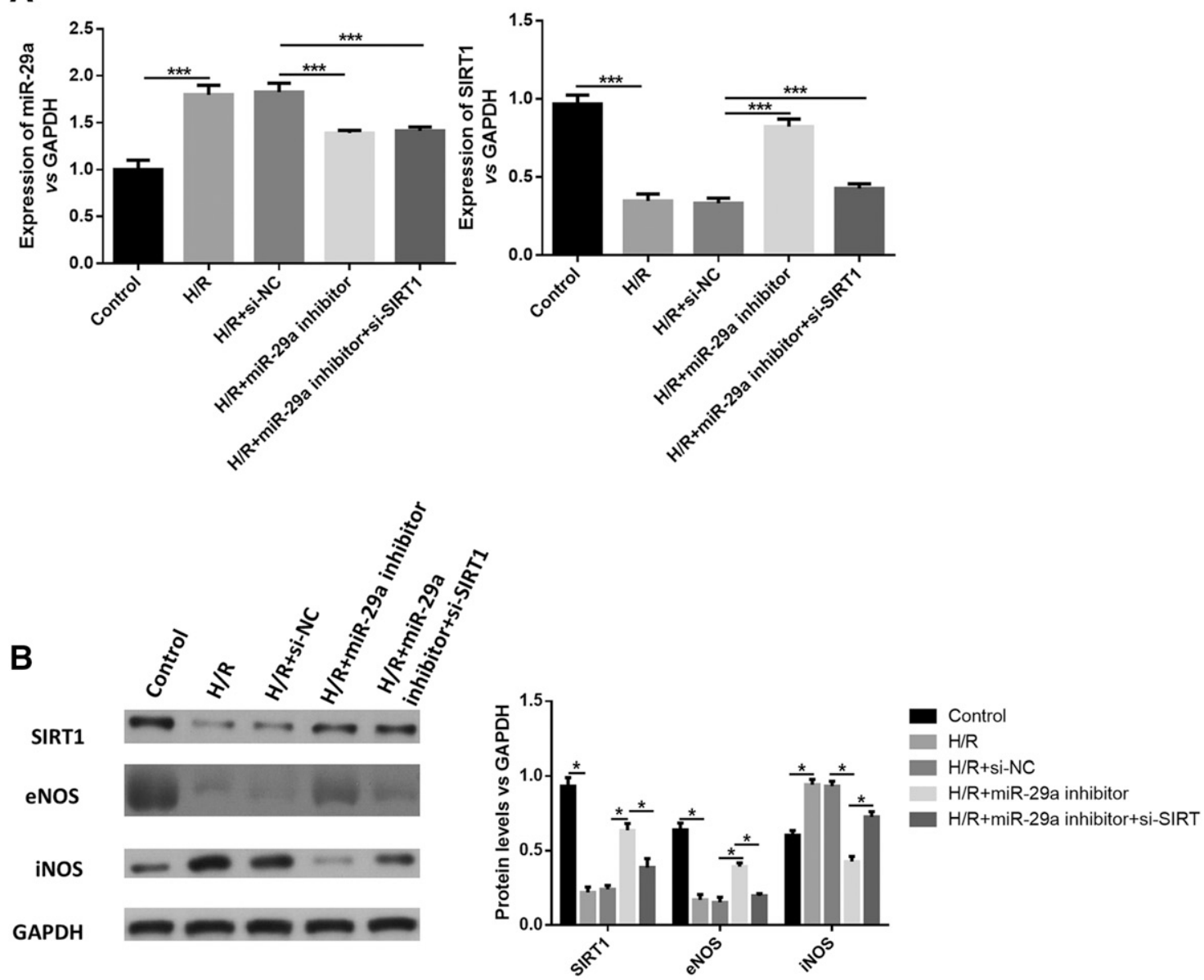

C
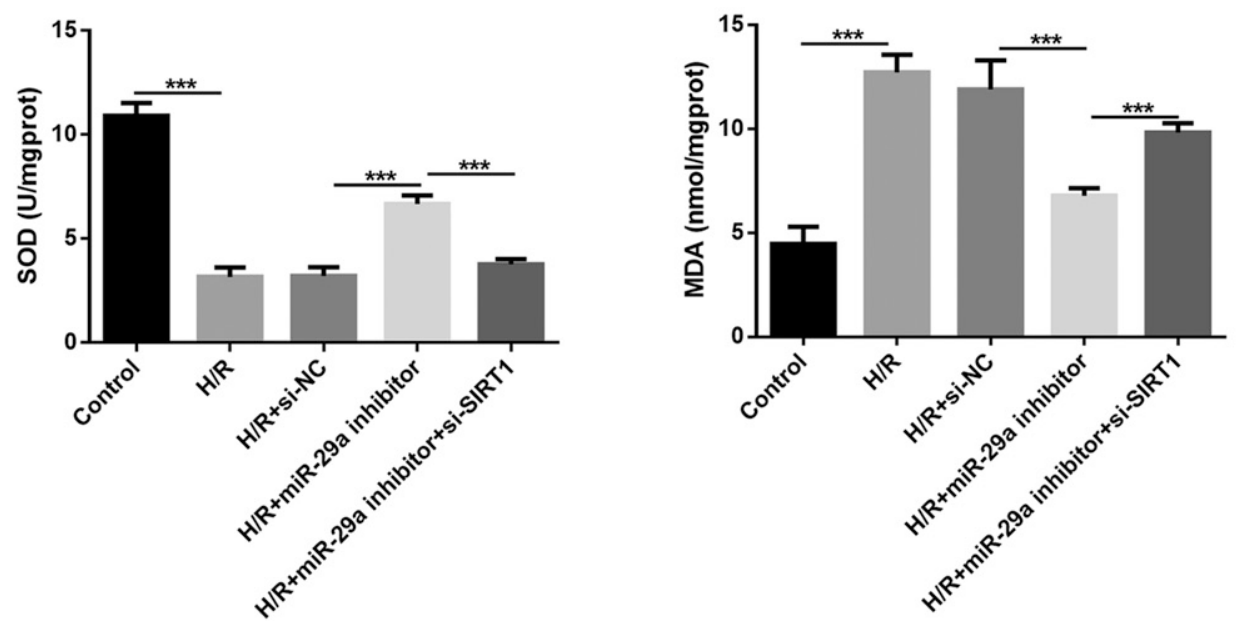

Fig. 4. Inhibition of miR-29a suppressed H/R-induced oxidative stress in H9c2 cells. (A) Expression of miR-29a and SIRT1 in different groups of cells. (B) Protein levels of SIRT1, eNOS, and iNOS in different groups of cells. (C) SOD and MDA levels in different groups of cells. $* * * P<0.001$; $* * P<0.01$.

In our study, we also found SIRT1 was down-regulated in I/R injury, and that the decrease of SIRT1 was correlated with I/R injury. We also have shown that miR-29a could improve I/R injury via targeting SIRT1.

The role of SIRT1 in I/R injury has been the subject of many studies. Hsu et al. (2011) demonstrated that SIRT1 could function as a protector for heart I/R injury through up-regulation of antioxidants and down-regulation of proapoptotic molecules. Yu et al. (2014) reported that the melatonin receptor could improve myocardial I/R injury by enhancing SIRT1 and reducing oxidative stress. Our study also has shown that the activation of SIRT1 by inhibition of mIR-29a inhibits oxidative stress and protects from I/R injury.

Up to now, few studies have focused on pyroptosis in I/R injury. Yang et al. (2014) reported that I/R injury could induce 
A

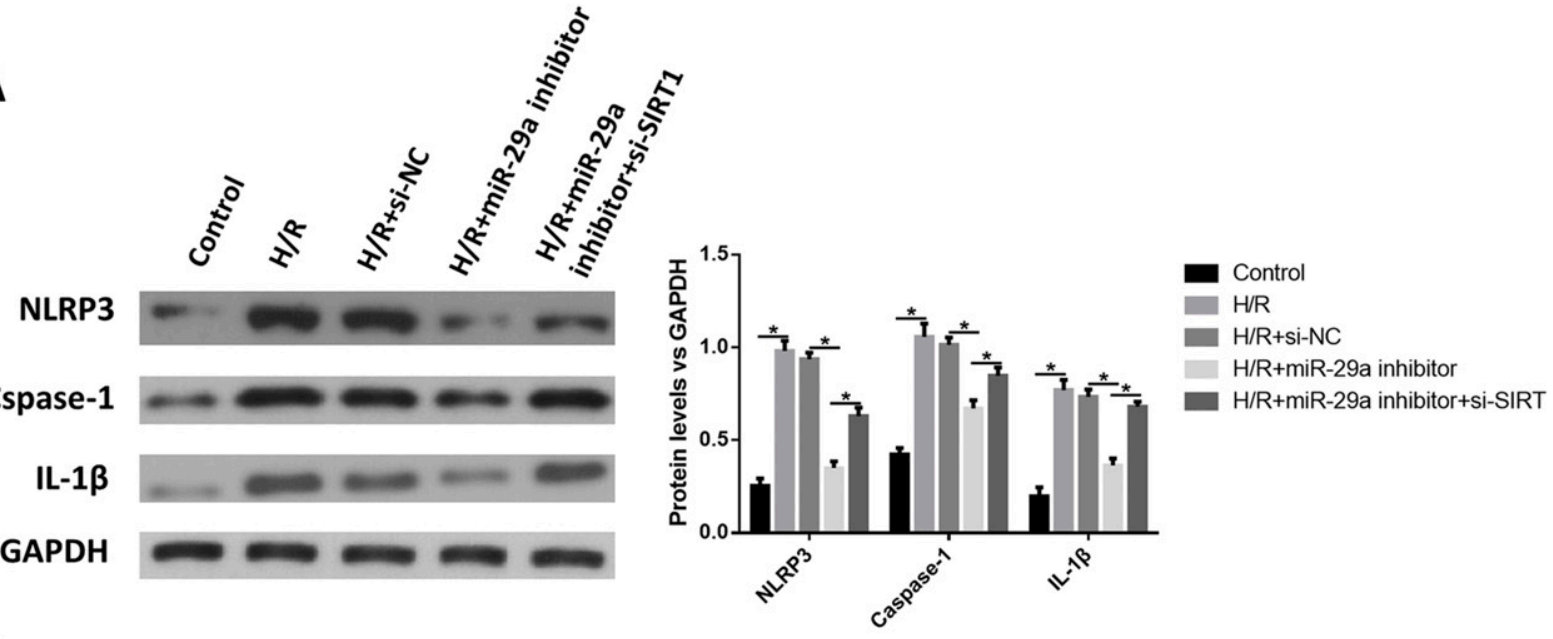

B
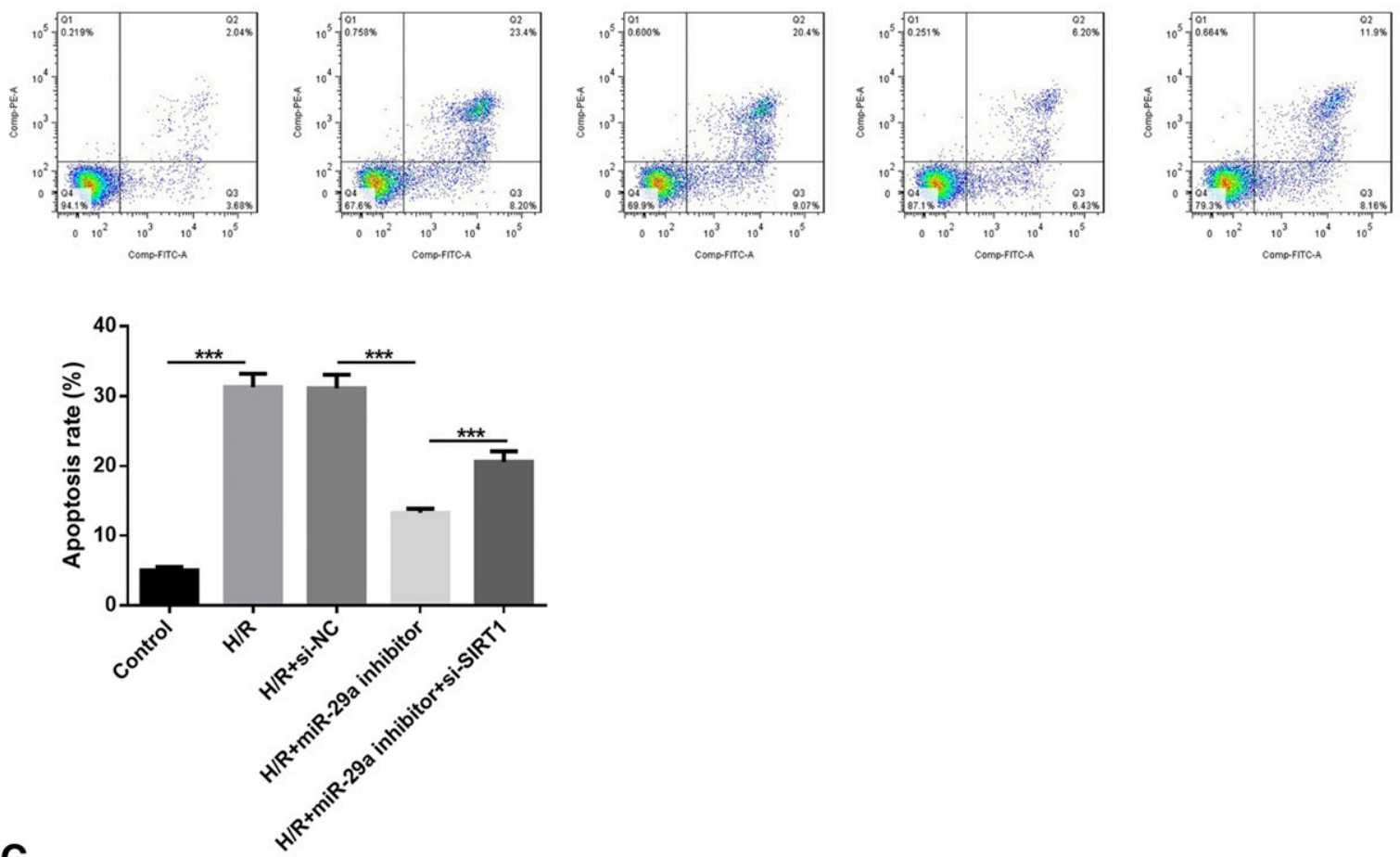

C

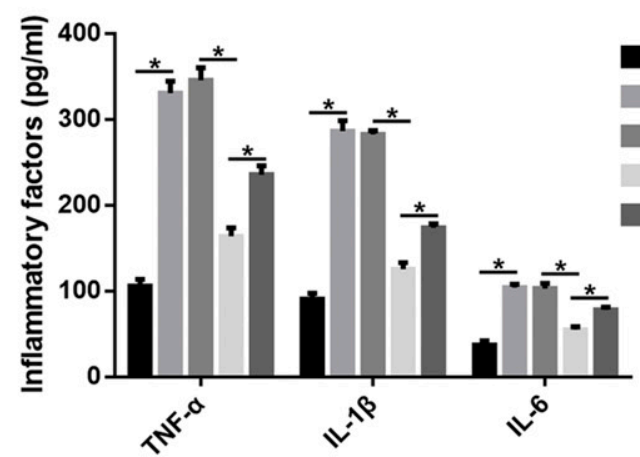

Control

$H / R$

$\mathrm{H} / \mathrm{R}+\mathrm{si}-\mathrm{NC}$

$H / R+m i R-29 a$ inhibitor

$H / R+m i R-29 a$ inhibitor+si-SIRT

. 5. Inhibition of miR-29a suppressed H/R-induced pyroptosis in H9c2 cells. (A) Protein levels of NLRP3, caspase-1, and IL-1 $\beta$ in different groups of cells. (B) Cell apoptosis in different groups of cells. (C) Levels of inflammatory factors in different groups of cells. $* * * P<0.001 ; * * P<0.01$.

renal tubule pyroptosis via the C/EBP homologous protein (CHOP)-caspase-11 pathway. To the contrary, the NLRP3mediated pyroptosis could facilitate the myocardial I/R injury
(Qiu et al., 2017). In this study, we showed for the first time that miR-29a could improve myocardial I/R injury through inhibition of pyroptosis. 


\section{Conclusion}

Inhibition of miR-29a improved myocardial I/R injury by targeting SIRT1 through suppressing oxidative stress and NLRP3-mediated pyroptosis.

\section{Authorship Contributions}

Participated in research design: Ding, Liu, L. Wang.

Conducted experiments: Ding, Liu, L. Wang.

Contributed new reagents or analytic tools: Ding, Liu, L. Wang, G. Wang, Zhu.

Performed data analysis: Ding, Liu, L. Wang.

Wrote or contributed to the writing of the manuscript: Ding, Liu,

L. Wang.

\section{References}

Anaya-Prado R, Toledo-Pereyra LH, Lentsch AB, and Ward PA (2002) Ischemia/ reperfusion injury. J Surg Res 105:248-258.

Chou CH, Shrestha S, Yang CD, Chang NW, Lin YL, Liao KW, Huang WC, Sun TH, Tu SJ, Lee WH, et al. (2018) miRTarBase update 2018: a resource for experimentally validated microRNA-target interactions. Nucleic Acids Res 46 (D1) D296-D302.

Corpas R, Revilla S, Ursulet S, Castro-Freire M, Kaliman P, Petegnief V, GiménezLlort L, Sarkis C, Pallàs M, and Sanfeliu C (2017) SIRT1 overexpression in mouse hippocampus induces cognitive enhancement through proteostatic and neurotrophic mechanisms. Mol Neurobiol 54:5604-5619.

Duan YF, Sun DL, Chen J, Zhu F, and An Y (2017) MicroRNA-29a/b/c targets iNOS and is involved in protective remote ischemic preconditioning in an ischemiareperfusion rat model of non-alcoholic fatty liver disease. Oncol Lett 13:1775-1782.

Fusco S, Maulucci G, and Pani G (2012) Sirt1: def-eating senescence? Cell Cycle 11 $4135-4146$.

Han Z, Cao J, Song D, Tian L, Chen K, Wang Y, Gao L, Yin Z, Fan Y, and Wang C (2014) Autophagy is involved in the cardioprotection effect of remote limb ischemic postconditioning on myocardial ischemia/reperfusion injury in normal mice, but not diabetic mice. PLoS One 9:e86838.

Hattori Y, Okamoto Y, Nagatsuka K, Takahashi R, Kalaria RN, Kinoshita M, and Ihara M (2015) SIRT1 attenuates severe ischemic damage by preserving cerebral blood flow. Neuroreport 26:113-117.

Hausenloy DJ and Yellon DM (2013) Myocardial ischemia-reperfusion injury: a neglected therapeutic target. J Clin Invest 123:92-100.

Hsu CP, Zhai P, Yamamoto T, Maejima Y, Matsushima S, Hariharan N, Shao D, Takagi H, Oka S, and Sadoshima J (2011) Silent information regulator 1 protects the heart from ischemia/reperfusion. Circulation 122:2170-2182.
Jayawardena TM, Egemnazarov B, Finch EA, Zhang L, Payne JA, Pandya K, Zhang Z, Rosenberg P, Mirotsou M, and Dzau VJ (2012) MicroRNA-mediated in vitro and in vivo direct reprogramming of cardiac fibroblasts to cardiomyocytes. Circ Res 110:1465-1473.

Lewis BP, Shih IH, Jones-Rhoades MW, Bartel DP, and Burge CB (2003) Prediction of mammalian microRNA targets. Cell 115:787-798.

Piper HM, Meuter K, and Schäfer C (2003) Cellular mechanisms of ischemiareperfusion injury. Ann Thorac Surg 75:S644-S648.

Qiu Z, Lei S, Zhao B, Wu Y, Su W, Liu M, Meng Q, Zhou B, Leng Y, and Xia ZY (2017) NLRP3 inflammasome activation-mediated pyroptosis aggravates myocardial ischemia/reperfusion injury in diabetic rats. Oxid Med Cell Longev 2017:9743280.

Quadri SM, Segall L, de Perrot M, Han B, Edwards V, Jones N, Waddell TK, Liu M, and Keshavjee S (2005) Caspase inhibition improves ischemia-reperfusion injury after lung transplantation. Am J Transplant 5:292-299.

Roderburg C, Urban GW, Bettermann K, Vucur M, Zimmermann H, Schmidt S, Janssen J, Koppe C, Knolle P, Castoldi M, et al. (2011) Micro-RNA profiling reveals a role for miR-29 in human and murine liver fibrosis. Hepatology 53:209-218.

Rupaimoole R and Slack FJ (2017) MicroRNA therapeutics: towards a new era for the management of cancer and other diseases. Nat Rev Drug Discov 16:203-222.

Sanderson TH, Reynolds CA, Kumar R, Przyklenk K, and Hüttemann M (2013) Molecular mechanisms of ischemia-reperfusion injury in brain: pivotal role of the mitochondrial membrane potential in reactive oxygen species generation. Mol Neurobiol 47:9-23.

Sassi Y, Avramopoulos P, Ramanujam D, Grüter L, Werfel S, Giosele S, Brunner AD Esfandyari D, Papadopoulou AS, De Strooper B, et al. (2017) Cardiac myocyte miR-29 promotes pathological remodeling of the heart by activating Wnt signaling. Nat Commun 8:1614.

Tang Y, Zheng J, Sun Y, Wu Z, Liu Z, and Huang G (2009) MicroRNA-1 regulates cardiomyocyte apoptosis by targeting Bcl-2. Int Heart $J$ 50:377-387.

Wang L, Niu X, Hu J, Xing H, Sun M, Wang J, Jian Q, and Yang H (2015) After myocardial ischemia-reperfusion, miR-29a, and Let7 could affect apoptosis through regulating IGF-1. BioMed Res Int 2015:245412.

Yang JR, Yao FH, Zhang JG, Ji ZY, Li KL, Zhan J, Tong YN, Lin LR, and He YN (2014) Ischemia-reperfusion induces renal tubule pyroptosis via the CHOPcaspase-11 pathway. Am J Physiol Renal Physiol 306:F75-F84.

Yu L, Sun Y, Cheng L, Jin Z, Yang Y, Zhai M, Pei H, Wang X, Zhang H, Meng Q, et al. (2014) Melatonin receptor-mediated protection against myocardial ischemia/ reperfusion injury: role of SIRT1. J Pineal Res 57:228-238.

Address correspondence to: Yaobin Zhu, Cardiovascular Surgery II, Beijing Children's Hospital, Capital Medical University, National Center for Children's Health, Beijing, 100045, People's Republic of China. E-mail: zzzyyy8607@ aliyun.com; or Guanggong Wang, Cardiovascular Surgery II, Beijing Children's Hospital, Capital Medical University, National Center for Children's Health, Beijing, 100045, People's Republic of China. Email: gfxk04@163.com 